\title{
Note on an "Alternant" with Factorial Elements
}

By ZIA-UDDIN, University of Edinburgh.

(Received May 22, 1933. Read June 3, 1933.)

$\S 1$. The alternant with powers of variables for its elements

$$
\left|\begin{array}{cccc}
a^{\alpha} & b^{\alpha} & \ldots & z^{a} \\
a^{\beta} & b^{\beta} & \ldots & z^{\beta} \\
\cdot & \cdot & \ldots & \cdot \\
\cdot & \cdot & \ldots & \cdot \\
a^{\omega} & b^{\omega} & \ldots & z^{\omega}
\end{array}\right|
$$

has often been investigated and its properties ${ }^{1}$ are well known. In the present note an " alternant" with factorial elements,

$$
\left(\begin{array}{ccccc}
a & b & c & \ldots & z \\
a & \beta & \gamma & \ldots & \omega
\end{array}\right) \equiv\left|\begin{array}{cccc}
a^{(\alpha)} & b^{(a)} & \ldots & z^{(a)} \\
a^{(\beta)} & b^{(\beta)} & \ldots & z^{(\beta)} \\
\cdot & \cdot & \ldots & \cdot \\
a^{(\omega)} & b^{(\omega)} & \ldots & z^{(\omega)}
\end{array}\right|
$$

will be discussed. Here $a, b, c, \ldots, z, a, \beta, \gamma, \ldots, \omega$ are integers such that

and

$$
\begin{aligned}
& a>b>c>\ldots>z \geqq 0, \\
& a>\beta>\gamma>\ldots>\omega \geqq 0, \\
& a \geqq \alpha, b \geqq \beta, \ldots, z \geqq \omega,
\end{aligned}
$$

$$
a^{(a)}=a(a-1)(a-2) \ldots(a-a+1),
$$

with the convention that $a^{(0)}=1,0^{(0)}=1$, and that, where negative indices occur (in the reductions of $\S 2$ ), the factorial is to be regarded as zero.

It is important in several investigations to show that a " factorial alternant" of this kind cannot vanish; in the present note, we prove

THeorem 1. The determinant

is always positive.

$$
\Delta \equiv\left(\begin{array}{lllll}
a & b & c & \ldots & z \\
a & \beta & \gamma & \ldots & \omega
\end{array}\right)
$$

1 See, for example, Muir's History of Determinants. 
$\S 2$. If $z>0$ the "alternant". $\Delta$ can be expressed as the sum, with positive coefficients, of similar factorial "alternants" with lower indices.

The expansion is

$$
\begin{aligned}
& \left(\begin{array}{rrrrr}
a & b & c & \ldots & z \\
a & \beta & \gamma & \ldots & \omega
\end{array}\right)=\left(\begin{array}{cccccc}
a-1 & b-1 & c-1 & \ldots & z-1 \\
a & \beta & \gamma & \ldots & \omega
\end{array}\right) \\
& +\Sigma a\left(\begin{array}{ccccccc}
a-1 & b-1 & c-1 & \ldots & z-1 \\
a-1 & \beta & \gamma & \ldots & \omega
\end{array}\right)+\Sigma a \beta\left(\begin{array}{ccccc}
a-1 & b-1 & c-1 & \ldots & z-1 \\
a-1 & \beta-1 & \gamma & \ldots & \omega
\end{array}\right) \\
& +\ldots+a \beta \gamma \\
& +\ldots\left(\begin{array}{ccccc}
a-1 & b-1 & \ldots & z-1 \\
a-1 & \beta-1 & \ldots & \omega-1
\end{array}\right) .
\end{aligned}
$$

For since $a^{(a)}=(a-1)^{(a)}+a(a-1)^{(a-1)}$, we may write every element of $\Delta$ as a binomial of this kind, and then expanding $\Delta$ as a sum of determinants with mononomial elements $(a-1)^{(a)}$ or $a(a-1)^{(a-1)}$, we obtain the above expansion.

The expansion of $\Delta$ can be written in operational form thus: If $A_{a}^{-1} f(a)=f(a-1)$, then

$\left(\begin{array}{llll}a & b & \ldots & z \\ a & \beta & \ldots & \omega\end{array}\right)=\left\{\left(1+a A_{a}^{-1}\right)\left(1+\beta A_{\beta}^{-1}\right) \ldots\left(1+\omega A_{\omega}^{-1}\right)\right\}\left(\begin{array}{cccc}a-1 & b-1 & \ldots & z-1 \\ \alpha & \beta & \ldots & \omega\end{array}\right)$, and by repeating the whole operation we can obtain successive expansions in terms of indices of lower and lower order. In such a reduction vanishing terms will in general occur, but we have to show that there always exist non-vanishing terms. It will be convenient now to write $a_{1}, a_{2}, \ldots, a_{n}$ for $a, b, \ldots, z$, and $a_{1}, a_{2}, \ldots, a_{n}$ for $\alpha, \beta, \ldots, \omega$.

§3. The following cases arise in the reduction.

(a) Some of the "alternants" will be vanishing. This will be the case when some $\alpha_{i}=a_{k}$.

(b) Some "alternants" will factorise into ones of lower order. This will be the case when some $a_{k}<a_{k-1}$.

For example if $a_{3}<a_{2}$, then

$$
\left(\begin{array}{lllll}
a_{1} & a_{2} & a_{3} & a_{4} & a_{5} \\
a_{1} & a_{2} & a_{3} & a_{4} & a_{5}
\end{array}\right)=\left(\begin{array}{ll}
a_{1} & a_{2} \\
a_{1} & a_{2}
\end{array}\right)\left(\begin{array}{lll}
a_{3} & a_{4} & a_{5} \\
a_{3} & a_{4} & a_{5}
\end{array}\right)
$$

for there will be found to be some zero elements above the diagonal permitting the factorisation. 
(c) In the particular case when

$$
a_{2}<a_{1}, a_{3}<a_{2}, \ldots a_{k}<a_{k-1}, \ldots \ldots a_{n}<a_{n-1},
$$

the factorisation is complete, and such an "alternant" is the product of its diagonal elements.

(d) An important special case of $\Delta$ is

$$
\left(\begin{array}{ccccc}
a_{1} & a_{2} & \ldots & a_{n-1} & a_{n} \\
n & n-1 & \ldots & 1 & 0
\end{array}\right)
$$

which is equal to the difference product of the elements $a_{n}$.

§4. It will now be shown that non-vanishing terms occur. For let the least of the differences $a_{2}-a_{1}, a_{3}-a_{2}, \ldots, a_{n}-a_{n-1}$, be $a_{k}-a_{k-1}$, or $p$ say; $p$ may be $0,1,2, \ldots$ Then in the reduction of $\Delta$ by the expansion formula of $\S 2$, there will occur, with positive coefficient, an "alternant" $\Delta^{\prime}$ with the $a$ 's all reduced by $p+1$, namely

$$
\Delta^{\prime \prime}=\left(\begin{array}{cccc}
a_{1}-p-1 & a_{2}-p-1 & \ldots & a_{n}-p-1 \\
a_{1} & a_{2} & \ldots & a_{n}
\end{array}\right) .
$$

Evidently $\Delta^{\prime}$, like $\Delta$, will satisfy the conditions $(A)$ of $\S 1$.

But, since now $a_{k}-p-1<a_{k-1}, \Delta^{\prime}$ factorises, in virtue of $\S 3(b)$, into

$\left(\begin{array}{cccc}a_{1}-p-1 & a_{2}-p-1 & a_{k-1}-p-1 \\ a_{1} & a_{2} & \ldots & a_{k-1}\end{array}\right)\left(\begin{array}{ccc}a_{k}-p-1 & \ldots & a_{n}-p-1 \\ a_{k} & \ldots & a_{n}\end{array}\right)$.

Each of these factors may be further reduced by finding the corresponding number " $p$ " for each of them and proceeding as above. We thus obtain "alternants" of lower and lower order, ${ }^{1}$ until, at last, we have to examine the type

$$
\left(\begin{array}{l}
a_{1} \\
a_{1}
\end{array}\right)
$$

where $a_{1} \geqq a_{1}, a_{1} \geqq 0, a_{1} \geqq 0$; but these, being ordinary factorials are obviously positive.

We have thus shown that, in the reduction of $\Delta$, vanishing terms and factorisable terms both occur. The latter give, on further reduction, vanishing terms and factorisable terms, and so on. In the final reduction in terms of "alternants" of order 1 (which are

1 The necessity for the convention $a(0)=1,0(0)=1$ is seen by examining the reduction of $\left(\begin{array}{ll}a & 1 \\ a & 0\end{array}\right)$ for the cases $a \geqq 1$ and $a=0$. 
ordinary factorials), we have, therefore, at least one, and in general many, positive terms or products of such terms with positive coefficients. Thus $\Delta$, being the sum of these terms, is positive; this proves the theorem.

$\S 5$. The non-vanishing property of $\Delta$ leads to an interesting result concerning the zeros of a polynomial and its derivatives.

Consider a polynomial of any degree containing only $n$ nonzero terms, including a constant term, say

$$
\phi(x)=A x^{a}+B x^{b}+\ldots+K .
$$

Its derivative of order $a$ is

$$
\phi^{(a)}(x)=A a^{(a)} x^{a-a}+B b^{(a)} x^{b-a}+\ldots
$$

If $\phi(x)$ and the $(n-1)$ derivatives $\phi^{(a)}(x), \phi^{(\beta)}(x), \ldots$ possessed a root in common, then a set of simultaneous equations

$$
\phi(x)=0, \quad \phi^{(a)}(x)=0, \quad \phi^{(\beta)}(x)=0, \ldots
$$

would be satisfied for some non-zero value of $x$. But the condition for this is that

$$
\Delta=\left(\begin{array}{lllll}
a & b & c & \ldots & 0 \\
a & \beta & \gamma & \ldots & 0
\end{array}\right)=0
$$

which is not the case, as we have already proved.

Hence we have

Theorem 2. If a polynomial, possessing a non-zero constant term, has $n$ terms, it cannot have a zero in common with more than $n-2$ of its derivatives.

It should be observed that this result depends only on the number of terms and is independent of the degree of the polynomial.

In conclusion, I wish to acknowledge my indebtedness to Dr A. C. Aitken for his kind guidance and advice during the preparation of this note. 